\title{
Investigation of the Nanostructures Formation in the Irradiated by $\gamma$-Quanta Single-Crystal Silicon with Ultrasonic Method
}

\author{
Turdali Khaydarov, Izida Khamidovna Abdukadirova, Yuriy Karimov \\ Institute of Nuclear Physics Academy of Sciences, Tashkent, Uzbekistan \\ Email: khaydarov.t@gmail.com
}

Received March 30, 2012; revised May 17, 2012; accepted May 31, 2012

\begin{abstract}
It's determined that a phasic dynamics of deformation strengthening of single-crystal silicon irradiated by $\gamma$-quanta (with energy $\sim 1.27 \mathrm{MeV}$ ) in the range of radiation absorbed doses from $10^{2}$ up to $10^{9}$ rad by the internal friction measurement with widely known ultrasonic resonance method. We have detected appearance the maximum on the dependence of internal friction $\left(\mathrm{Q}^{-1}\right)$ from dose at $5 \times 10^{5} \mathrm{rad}$ in the specimens of $\mathrm{p}$-Si with a density of the dislocations more than $10^{3} \mathrm{~cm}^{-2}$. The instability of the dislocation structures has been established in the range of doses from $10^{6}$ up to $10^{9}$ $\mathrm{rad}$, due to the formation and accumulation in the crystal lattice of the point like and the continuous radiation defects. On the time dependence of $\mathrm{Q}^{-1}(\mathrm{t})$ per 1.5 - 2 hours after irradiation, the maximum has been established which position depends on dose of radiation. The monotonic decrease of $\mathrm{Q}^{-1}(\mathrm{t})$ dependence was observed at the increase of the observation time after stopping of the specimen irradiation, which is connected with decreasing of the radiation defects densities as the result of their annihilation.
\end{abstract}

Keywords: Internal Friction; Density of Dislocations; Si; Radiation Defects; Annihilation

\section{Introduction}

Some physical properties of materials are connected with their dislocated structure. Dislocated structures of materials can be created by the plastic deformation or under influence of the gamma irradiation [1]. Radiation defects are closely connected with the dislocation strengthening. Mechanisms of the deformation strengthening and of the formation of fragmentary dislocation structures in the metals have been theoretically discussed in the previous studies [2,3].

Although the processes of the multiplication, of the diffusion, and of the annihilation of dislocations in the metals and semiconductors have been considered in [1-9], but the main goal of majority investigations consist in a possibility of analysis those effects from the kinetic positions.

However, the dynamic processes of dislocations and of radiations defects in the materials at interruption in the irradiation still are not well understood. Information concerning this will be allows us to predict a resource of the semiconducting material, and a time behaviour of curves of deformation strengthening, and of specific structures at the mechanical fatigue of crystals. In this connection it's interesting the investigation of dynamic of radiation defects in the semiconductor after stopping of influence of the radiation.

In present paper, the dynamic of radiation defects into single-crystals silicon are investigated in the range of doses from $10^{2}$ rad up to $10^{9} \mathrm{rad}$ by the internal friction measurement with the ultrasonic resonance method [10], step by step.

\section{Methods and Objects of Investigation}

Measurements of internal friction $\left(\mathrm{Q}^{-1}\right)$ of the singlecrystal silicon have been made with the ultrasonic resonance method before and after irradiation. The silicon specimens with gauges of $35 \mathrm{~mm}$ length, $3 \mathrm{~mm}$ width, $1 \mathrm{~mm}$ thickness, and of $28.7 \mathrm{~mm}$ length, $4.1 \mathrm{~mm}$ width, $1.2 \mathrm{~mm}$ thickness, and of $20 \mathrm{~mm}$ length, $1 \mathrm{~mm}$ width, and $1 \mathrm{~mm}$ thickness were used. The values of intrinsic resonances of the longitudinal vibrations of specimens were in the frequency interval from $50 \mathrm{kHz}$ to $300 \mathrm{kHz}$. The amplitude of input signal was approximately $7 \mathrm{~V}$ to $7.2 \mathrm{~V}$ for launching of vibration into the specimen. Method of fixing of the specimen is shown on insertion (a) (see Figure 1). Measurements were carried out at a room temperature $\left(\sim 25^{\circ} \mathrm{C}\right)$. After stopping of influence of the radiation during 10 minutes, the specimen 


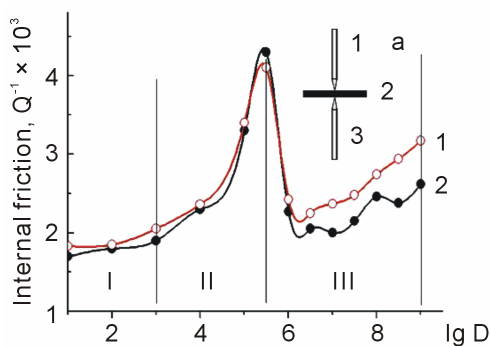

Figure 1. Dependence the internal friction of single-crystal of the silicon from the radiation absorbed dose. Curve \#1 is measured on frequency $149 \mathrm{kHz}$, curve \#2: $69 \mathrm{kHz}$. I: first stage, II: second stage, III: third stage. On inset are shown: a: method of fixing of specimen where 1 is transfer acoustic line and 3: receive acoustic line. 2 : specimen.

was set up between acoustic lines 1 and 3. From that time the measurement of the internal friction $\left(\mathrm{Q}^{-1}\right)$ was started. Value of the internal friction was calculated with the following equation $\mathrm{Q}^{-1}=\Delta \mathrm{f} / \mathrm{f}_{\mathrm{o}}$ (root-mean-square error $8 \%$ ).

The bandwidth $(\Delta f)$ is the width of the range of frequencies where the signal is 0.707 of the maximum signal amplitude, $f_{o}$ is the resonant frequency. The basic resonant frequencies $\mathrm{f}=69 \mathrm{kHz}$ and $\mathrm{f}=149 \mathrm{kHz}$ were used.

The alloyed single-crystals of silicon of the p-type conductivity with density of dislocations more than $10^{3}$ $\mathrm{cm}^{-2}$ and with specific resistance $10 \mathrm{ohm}$ meter and 50 ohm meter were used as initial specimens. Specimens were irradiated into $\gamma$-channels of ${ }^{60} \mathrm{Co}$ gamma source with range of dose rate from $10 \mathrm{rad}$ per sec to $500 \mathrm{rad}$ per sec. Above mentioned intensity of gamma rays quite enough for study of a dynamic of radiations defects in the single-crystal silicon where a slight concentration of defects exerts important influence on the acoustic wave absorption

\section{Results and Discussion}

\subsection{The Dependence of Internal Friction from Dose}

The irradiation was used in order that to create a defective structure evenly in all volume of thin specimen. Then we had observed the time-dependent behaviour of internal friction. Dependence internal friction from the irradiation dose $\left(Q^{-1}(D)\right)$ is shown on Figure 1. It's can to divide on three stages: I: first stage where the internal friction essentially not changed with an increase of the radiation absorbed dose (D) from 10 to $10^{3} \mathrm{rad}$; II: second stage where the internal friction reached maximum with an increase of the D from $10^{3}$ to $5 \times 10^{5} \mathrm{rad}$; IIIthird stage where the internal friction dropped down at D $=10^{6} \mathrm{rad}$ and further it is slowly rising with an increase of the D from $10^{6}$ to $10^{9} \mathrm{rad}$.
First value of $\mathrm{Q}^{-1}$ (see Figure 1) depends from dislocation density that initial specimen contains. The more initial dislocation density, the more value $\mathrm{Q}^{-1}$.

Dependence the internal friction from of the radiation absorbed dose $Q^{-1}(D)$ (shown on Figure 1) and time dependence of internal friction $\mathrm{Q}^{-1}(\mathrm{t})$ (shown on Figure 2) were observed in the specimens that have density of dislocations $\rho \geq 10^{3} \mathrm{~cm}^{-2}$.

$\mathrm{Q}^{-1}(\mathrm{D})$ and $\mathrm{Q}^{-1}(\mathrm{t})$ dependences didn't observed in the specimens if they preliminary anneal and then irradiate.

Dependence $\mathrm{Q}^{-1}(\mathrm{D})$ obtained as result of irradiate (see Figure 1) correspond to such process of deformation strengthening that observed in the silicon at the temperature around $750^{\circ} \mathrm{C}[11,12]$.

We suppose that the generation of the deformation strengthening in the silicon at temperature around $750^{\circ} \mathrm{C}$ and a similar process at room temperature in the irradiated specimen of the silicon are connected with a mechanism of formation of the dislocation loops. This dependence $Q^{-1}(D)$ demonstrate that there are three stage of the deformation strengthening in the silicon with an increase of the radiation absorbed dose. As known the internal friction $Q^{-1}$ is very sensitive to the little changes of the location of the pinning point of dislocations and it is the measure of strengthening of specimens [1,4,5]. Seemingly, anew arising dislocations bring in the dependence $\mathrm{Q}^{-1}(\mathrm{D})$ the basic contribution. For example the radiation defects didn't exert important influence on the $\mathrm{Q}^{-1}$ at the first stage of irradiation (until $10^{3} \mathrm{rad}$ ) but they make a flat slope of the value of internal friction at the dependence (D) that connected with a speed of formation of dislocation loops. We suppose that growth of quantity of unsteady dislocations in the interval of doses from $10^{3}$ to $5 \times 10^{5}$ to facilitate progress a new dislocation loops which found up the dislocation band structure (see Figure 1, II: second stage). Whereas size of loops particularly not depends from dose of radiation [13], the second stage II of the curve $Q^{-1}(D)$ (see Figure 1) demonstrate the generation of dislocation cell structure and as result the irradiated specimen have the radiation strengthening. Dose of radiation $5 \times 10^{5}$ rad generate the critical density of dislocation loops that create favourable conditions for forming free from defects channels [2,3] and thereby bring to sequence transition from dislocation structures to annihilation structures. Whereas the radiation doses modify the density of loops in accordance with law [2]: $\rho$ $\infty \mathrm{D}^{\mathrm{n}}$, where $(\mathrm{n}=1 / 2-2 / 3)$. The parabolic dependence $\mathrm{Q}^{-1}(\mathrm{D})$ at I and II stages of radiation strengthening demonstrate that in this interval of dose: $\mathrm{Q}^{-1} \infty \rho^{2 / 3}$.

However the behavior of radiation strengthening corresponds to similarly process in the single crystal of metal at III stage.

Decreasing of the value of the internal friction is shown in Figure 1 at the radiation doses over $5 \times 10^{5} \mathrm{rad}$ 
as result of the strengthening of the specimen. Such drop of the value $\mathrm{Q}^{-1}$ is connected with an increase quantity loops that have density $\rho \sim 10^{9}-10^{10} \mathrm{~cm}^{-2}[2,14]$ at dose over $5 \times 10^{5} \mathrm{rad}$.

\subsection{The Time Dependence of Internal Friction}

The plastic deformation of irradiated crystals has a feature that connected not only with a strengthening influence of radiation defects but and with an unstable nature of behavior of plastic deformation [2].

As known, the energy of dislocation have approximately value $\sim 10 \times 10^{-19}$ joule (or around $6 \mathrm{eV}$ ) at the distance between atoms along of the dislocation line $(\sim 2.5 \AA)$. For single crystal silicon the amount of energy of such dislocation is $19 \mathrm{eV}$ [12]. There is a strong probability that such high energy will increase the annihilation of the dislocations. Also, it has been reported [2], the generation of cell structure is connected with an action of a few systems of glides at second and third stage of deformation strengthening during the process of plastic deformation.

Two competitive process are developing at the interval of doses over $5 \times 10^{5} \mathrm{rad}$ : on the one hand with generation of dislocation cell structure, on the other hand the annihilation of dislocation loops that create boundaries of extensive formations (channels without dislocation) [2].

Though both of the processes are developing at the irradiation but their equilibrium state depend from dose of radiation and temperature.

Probably process of annihilation of dislocation loops prevail over process of formation of cell structure and internal friction $\mathrm{Q}^{-1}$ has moderate growth into interval of dose from $10^{6}$ to $10^{9} \mathrm{rad}$.

When the radiation absorbed dose of the initial specimen had a value in the range of doses from $10^{6} \mathrm{rad}$ up to $10^{9} \mathrm{rad}$, the internal friction increased till its maximum value during 1.5 - 2 hours after stopping of the irradiation (see Figure 2).

It was as result probably of the intensive progress of process of annihilation of dislocation loops, which has

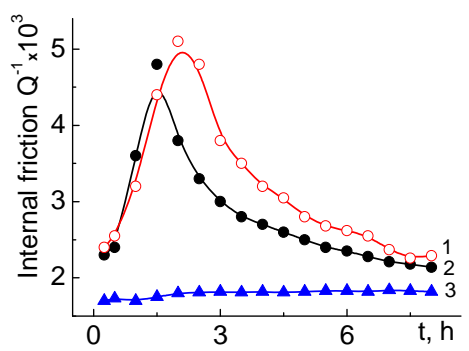

Figure 2. Time dependence of internal friction of the singlecrystal silicon: 1: at value of radiation absorbed dose $10^{9}$ rad; 2: at value of radiation absorbed dose $10^{8}$ rad; 3 : without of the irradiation. cooperative nature of the evolution.

It should be noted that value of internal friction was changing through $7-8$ hours and even through twentyfour hours from starting point of observation.

The process of annihilation has the further development in a random way as result of a sequential activation of dislocation kinks within a boundary microstructure by fields of internal tensions and process of annihilation steadily tend to its equilibrium value. Such long process of annihilation is connected probably with more late a diffusion of delayed defects to dislocations and to boundaries of cells.

\section{Conclusions}

It has been demonstrated in the present work that received dependences of internal friction from the radiation absorbed dose $\left(Q^{-1}(D)\right)$ have characterized the staging step of the deformation strengthening of alloyed single-crystal silicon.

We have detected appearance maximum on the dependence of internal friction from dose $Q^{-1}(D)$ at $5 \times$ $10^{5} \mathrm{rad}$ in the specimens of single crystal of silicon with density of dislocations more than $10^{3} \mathrm{~cm}^{-2}$.

It is the critical dose that characterizes the yield limit when the cell structures and the defect-free channels are formed into specimen. The generation of defect-free channels depend from energy and properties of defects which interact with each other in the structure of irradiated silicon.

The activity of dislocation loops is changing with a growth of the radiation absorbed dose. Destination of maximum on the temporal dependence of the internal friction $\left(\mathrm{Q}^{-1}(\mathrm{t})\right)$ depend from the radiation absorbed dose by specimen (i.e. the more radiation absorbed dose, the later arise maximum $\left.\mathrm{Q}^{-1}(\mathrm{t})\right) \cdot \mathrm{Q}^{-1}(\mathrm{D})$ and $\mathrm{Q}^{-1}(\mathrm{t})$ dependences didn't observed in the specimens if they preliminary anneal and then irradiate.

The monotonic decrease of $\mathrm{Q}^{-1}(\mathrm{t})$ dependence was observed at the increase of the observation time after stopping of the specimen irradiation, which is connected with decreasing of the radiation defects densities as the result of their annihilation. The long process of annihilation is connected with more late a diffusion of delayed defects to dislocations and to boundaries of cells.

\section{Acknowledgements}

The authors are thankful to Prof. M. Karimovu for important remark and R. Khamraevoy and Sh. Makhmudovu for concession of samples.

\section{REFERENCES}

[1] W. Mason, "Physical Acoustics, Principles and Methods," 
The Effect of Imperfections, Vol. III, Part A, New York, 1966, p. 578.

[2] G. A. Malygin, "Dislocation Self-Organization Processes and Crystal Plasticity," Physics-Uspekhi, Vol. 169, No. 9, 1999, pp. 979-1010.

[3] G. A. Malygin, "Analysis of the Factors That Cause Unstable Deformation and Loss of Plasticity in Neutron-Irradiated Copper," Physics of the Solid State, Vol. 47, No. 4, 2005, pp. 632-637.

[4] L. J. Teutonico, F, V. Granato and K. Lucke, "Theory of the Thermal Breakaway of a Pinned Dislocation Line with Application to Damping Phenomena," Journal of Applied Physics, Vol. 35, 1964, p. 220.

[5] Y. Kawasaki, "Cell Structures in Deformed Copper Single Crystals," Journal of the Physical Society of Japan, Vol. 36, 1974, pp. 142-148.

[6] T. Khaydarov and I. Khidirov, "The Time-Dependent Internal Friction in Titanium Nitride," Metallofizika, Vol. 13, No. 11, 1991, pp. 120-122.

[7] Y. Hiki, "Internal Friction of Lead. I," Journal of the Physical Society of Japan, Vol. 13, 1958, p. 1138.

[8] A. G. Mart'yanov, S. M. Shandarov and R. V. Litvinov, "Interaction of Light Waves on a Reflecting Holographic
Grating in Cubic Photorefractive Crystals," Physics of the Solid State, Vol. 44, No. 6, 2002, pp. 1050-1054.

[9] I. Khidirov, D. I. Sotvoldiev, S. Z. Zaynobiddinov and K. O. Baxtibaev, "Oscillating Time Dependence of Internal Friction in $\gamma$-Irradiated Nonstoichiometric Titanium Carbide and Hydrogen Influence on It," Papers of the Academy of Sciences of $R U z$, Vol. 6, 2008, p. 67.

[10] V. M. Baranov, "Acoustic Measurements in the Nuclear Power Engineering," Energoatomizdat, Moscow, 1990, p. 320.

[11] M. Victoria, N. Baluc, C. Bailat, Y. Dai, M. I. Luppo, R. Shaublin and B. N. Singh, "The Microstructure and Associated Tensile Properties of Irradiated fcc and bcc Metals," Journal of Nuclear Materials, Vol. 276, No. 1-3, 2000, pp. 114-122.

[12] J. V. Sharp, "Deformation of Neutron-Irradiated Copper Single Crystals," Philosophical Magazine, Vol. 16, No. 139, 1967, pp. 77-96.

[13] A. R. Velihanov, "Electroplasticity of Undoped and Doped Silicon," Physics and Astronomy, Vol. 44, No. 2, 2010, pp. 137-140.

[14] K. V. Ravi, "Imperfections and Impurities in Semiconductor Silicon," Wiley, New York, Toronto, 1981, p. 472. 